\title{
Inventing Less, Reusing More, and Adding Intelligence to Business Process Modeling
}

\author{
Lucinéia H. Thom ${ }^{1}$, Manfred Reichert ${ }^{1}$, Carolina M. Chiao ${ }^{2}$, \\ Cirano Iochpe ${ }^{2}$, and Guillermo N. Hess ${ }^{2}$ \\ ${ }^{1}$ Institute of Databases and Information Systems, \\ Ulm D-89069 - Ulm, Germany \\ \{lucineia.thom, manfred.reichert\}@uni-ulm.de \\ ${ }^{2}$ Institute of Informatics, Federal University of Rio Grande do Sul, \\ Av. Bento Gonçalves, 9500, 91501-970 - Porto Alegre, RS, Brazil \\ \{cchiao, ciochpe, hess\} @inf.ufrgs.br
}

\begin{abstract}
Recently, a variety of workflow patterns has been proposed focusing on specific aspects like control flow, data flow, and resource assignments. Though these patterns are relevant for implementing Business Process Modeling (BPM) tools and for evaluating the expressiveness of BPM languages, they do not contribute to reduce redundant specifications of recurrent business functions when modeling business processes. Furthermore, contemporary BPM tools do not support process designers in defining, querying, and reusing activity patterns as building blocks for process modeling. Related to these problems this paper proposes a set of activity patterns, evidences their practical relevance, and introduces a BPM tool for the modeling of business processes based on the reuse of these activity patterns. Altogether our approach fosters reuse of business function specifications and helps to improve the quality and comparability of business process models.
\end{abstract}

Keywords: business function, activity pattern, business process modeling.

\section{Introduction}

Business processes help organizations to better align their business goals with the needs of their customers; i.e., business processes constitute the glue between the strategic and the operational level of the organization [1]. To stay competitive in their market, organizations and companies are increasingly interested in improving the quality and the efficiency of their business processes as well as their interactions with customers and business partners [2].

Process-aware information systems (PAISs) offer promising perspectives to realize these goals, and a growing interest in aligning information systems (IS) in a processoriented way can be observed. To allow for more flexibility, PAISs introduce an additional layer when compared to traditional information systems, which provides an explicit description of the process logic. In general, this logic is represented as a process model which can be created using a business process modeling (BPM) tool. 
The introduction of PAISs and the adoption of BPM tools offer promising perspectives: (a) companies obtain a precise and unambiguous description of their business processes; (b) the definition of new business processes and new process models respectively can be speed up significantly; (c) the work between different actors can be coordinated more effectively; (d) real-time data about in-progress processes can be gathered and visualized; and (e) business processes can be standardized. Through Web Service technology, in addition, the benefits of BPM can be applied to crossorganizational business processes as well [3], [4].

Business processes comprise different business functions with specific and welldefined semantics, which can be considered as self-contained building blocks. Generally, a particular business function may occur several times within one or multiple business process models [3]. As example consider the process for approving the contents of a newsletter (cf. Fig 1). This simple business process includes three activities with following order: (a) The author sends a request for approving the article to be published to the editor of the current newsletter edition. (b) The editor reviews the contents of the article; she either approves it or requests changes from the author. (c) If the newsletter article is not sent to the editor after a certain period of time, the author will receive a respective notification. Obviously, this process comprises business functions with generic semantics, which recur in numerous business processes: Task Execution Request (a), Approval (b), and Notification (c). As we will discuss later such recurrent business functions can be described in terms of activity patterns in order to foster their reuse and to improve the quality and comparability of business process models.

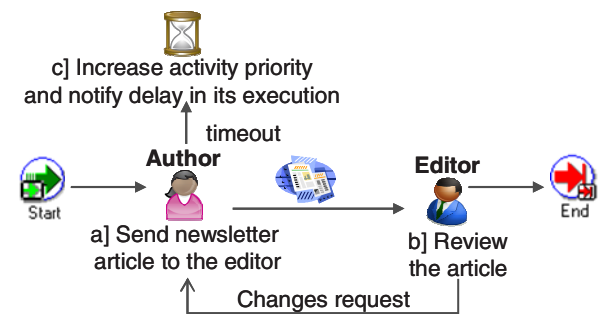

Fig. 1. Approval process for newsletter content

Recently a variety of workflow patterns has been proposed focusing on different process aspects like control flow [5], resource assignments [6], data flow [7], exception handling [8], domain-specific ontologies [9], service interactions [10], process change [11,30], and process compliance [12]. Though all these patterns are useful for implementing BPM tools and for evaluating the expressiveness of BPM languages, they do not contribute to avoid redundant specifications of recurrent business functions when modeling business processes. Consequently, business process design becomes inefficient, complex and time-consuming when being confronted with a large collection of business functions and business process models. To our best knowledge there exists no comprehensive work evidencing the existence of activity patterns for defining such recurrent business functions within business process models. Furthermore, no efforts have been spent on investigating the need, benefits and completeness 
of activity patterns with respect to business process modeling. Finally, contemporary BPM tools like Intalio, ARIS Toolset and WBI Modeler do not support process designers in defining, querying and reusing activity patterns as building blocks for business process modeling.

Related to these problems we proposed a set of seven workflow activity patterns and corresponding design choices (i.e., pattern variants) in previous work [3], [13]. Each of these activity patterns captures a recurrent business function as we can find it in numerous business processes like the one shown in Fig. 1. Combined with existing control flow patterns (e.g., sequence, multi instance activity), these activity patterns are suited to design a large variety of process models in different domains.

In this paper we briefly report on the results of an empirical study in which we analyze the relative frequency of activity patterns in a collection of 214 real-world process models from domains like quality management, software access control, and electronic change management. For selected process categories, we further discuss results of an additional analysis in which we investigate the frequency of co- occurring activity patterns. The results of this second analysis are also utilized for developing a BPM tool, which shall foster the modeling of business processes based on the reuse of activity patterns. Given some additional information about the kind of process to be designed, the results of our analysis can be further used by this tool to suggest a ranking of the activity patterns suited best to succeed the last pattern applied during process modeling.

One of the basic pillars of this BPM tool constitutes an ontology to describe the activity patterns. Particularly, this ontology allows to store and retrieve the patterns (together with their properties and constraints) during process modeling. To obtain machine-readable specifications, we suggest using a standard ontology language (e.g., OWL). Based on this, any BPM model based on the activity patterns can be easily and automatically transformed to conventional process modeling notations (e.g., BPMN) or languages (e.g., WS-BPEL, XPDL). Finally, using an ontology allows to make the relationships between the different activity patterns more explicit, which provides useful information for process designers.

The remainder of this paper is organized as follows: Section 2 gives an overview of workflow activity patterns. Exemplarily, we discuss basic principles taking the $A p$ proval Pattern. Section 3 presents the results of our empirical study in which we investigate the occurrence and relevance of activity patterns in practice by analyzing 214 real-world process models. Section 4 sketches our process modeling tool and discusses how the user interacts with it. In this context we also describe the ontology representing the patterns in detail. Section 5 discusses related work and Section 6 concludes with a summary and outlook.

\section{Workflow Activity Patterns}

In this paper we use the term Workflow Activity Pattern (WAP or activity pattern for short) to refer to the description of a recurrent business function as it frequently occurs in business process models. Examples include notification, decision, and approval. Initially, we derived seven activity patterns based on an extensive literature study [3], [13]. Table 1 gives an overview of these patterns. Generally, these activity 
patterns are close to the abstraction level or vocabulary used within an organization. This, in turn, fosters their reuse when modeling business processes, and therefore contributes to more standardized and better comparable business process models.

Each activity pattern is characterized by a short description, an example, a description of the problem context in which it can be applied, and relevant issues. Our framework considers additional attributes as well like design choices (pattern variants), related patterns and pattern implementation. However, these attributes are outside the scope of this paper and are omitted here. Fig. 2 gives a simple example of the description for the APPROVAL activity pattern (here restricted to a particular pattern variant, namely single approval; i.e., approval is required from exactly one role).

Table 1. Selected variants of activity patterns representing business functions

\begin{tabular}{|l|l|}
\hline WAP - Name & Description \\
\hline $\begin{array}{l}\text { WAP I: } \\
\text { Approval }\end{array}$ & $\begin{array}{l}\text { An object (e.g., a business document) has to be approved by one or more organiza- } \\
\text { tional roles. }\end{array}$ \\
\hline $\begin{array}{l}\text { WAP II: } \\
\text { Question-Response }\end{array}$ & $\begin{array}{l}\text { A question which emerges during process enactment has to be answered. This pattern } \\
\text { allows to formulate the question, to identify the organizational role(s) who shall } \\
\text { answer it, to send the question to the respective role(s), and to wait for the response(s) } \\
\text { (single-question-response) }\end{array}$ \\
\hline $\begin{array}{l}\text { WAP III: } \\
\text { Unidirectional Performative }\end{array}$ & $\begin{array}{l}\text { A sender requests the execution of a particular task from another process participant. } \\
\text { The sender continues process execution immediately after having sent the request for } \\
\text { performing the activity. }\end{array}$ \\
\hline $\begin{array}{l}\text { WAP IV: } \\
\text { Bi-directional Performative }\end{array}$ & $\begin{array}{l}\text { A sender requests the execution of a particular task from another process actor. The } \\
\text { sender waits until this actor notifies him that the requested task has been performed. }\end{array}$ \\
\hline $\begin{array}{l}\text { WAP V: } \\
\text { Notification }\end{array}$ & $\begin{array}{l}\text { The status or result of an activity execution is communicated to one or more process } \\
\text { participants. }\end{array}$ \\
\hline $\begin{array}{l}\text { WAP VI: } \\
\text { Informative }\end{array}$ & $\begin{array}{l}\text { An actor requests certain information from a process participant. He continues process } \\
\text { execution after having received the requested information. }\end{array}$ \\
\hline $\begin{array}{l}\text { WAP VII: } \\
\text { Decision }\end{array}$ & $\begin{array}{l}\text { This pattern can be used to represent a decision activity in the flow with different } \\
\text { connectors to subsequent execution branches. Those branches will be selected for } \\
\text { execution whose transition conditions evaluate to true. }\end{array}$ \\
\hline
\end{tabular}

\section{WAP1: APPROVAL (SIMPLIFIED VARIANT)}

Description: An object (e.g., a business document) has to be approved. Depending on the given context the approval is requested from one or multiple organizational roles. In the latter case, approval is done either sequentially or in parallel. Example: In an electronic change management process, a particular change request has to be approved concurrently by all roles concerned by the change. If one of these roles rejects the requested change, it will be not approved.

Problem: During the execution of a business process, object approval by one or multiple organizational roles is required before proceeding with the flow of control.

Issues:

a) The approval activity is executed only once by a particular organizational role.

b) Approval by multiple roles is needed for processes running in flat and decentralized organizations.

c) Final decision can be made manually (i.e., by a user) or automatically according to some rules.

Solution: The below process fragment illustrates the activity pattern for single approval using BPMN notation; here an organizational role reviewer performs a document review either resulting in approval or disapproval.
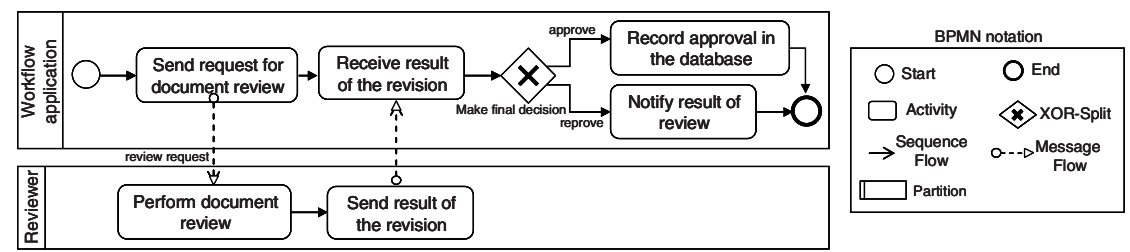

Fig. 2. Approval pattern 


\section{Evidencing the Existence and Relevance of Activity Patterns in Practice}

To identify activity patterns in real workflow applications we analyzed 214 process models. These process models have been modeled either with the Oracle Builder tool or an UML modeler. Our analysis has been based on process models instead of event logs, since we consider the semantics and the context of process activities as being fundamental for identifying activity patterns. Altogether, the analyzed process models stem from 13 different organizations - private as well as governmental ones - and are related to different applications like Total Quality Management (TQM), software access control, document management, help desk services, user feedback, document approval, and electronic change management. In all organizations the respective process models have been operationalized, i.e. they were supported by a PAIS. Table 2 summarizes information about involved organizations and analyzed process models.

Table 2. Characteristics of the analyzed process models

\begin{tabular}{|l|l|l|l|}
\hline $\begin{array}{l}\text { Size and Number of } \\
\text { Companies }\end{array}$ & $\begin{array}{l}\text { Kind of Decision- } \\
\text { making }\end{array}$ & Examples of Analyzed Process Models & $\begin{array}{l}\text { Number of } \\
\text { Analyzed Models }\end{array}$ \\
\hline $1 \times$ small & Decentralized & Management of Internal Activities & 17 \\
\hline $1 \times$ large & Decentralized & TQM; Management of Activities & 11 \\
\hline $6 \times$ large & Centralized & $\begin{array}{l}\text { TQM; Control of Software Access; } \\
\text { Document Management }\end{array}$ & 133 \\
\hline $4 \times$ large & No information available & $\begin{array}{l}\text { Help Desk Services, User Feedback; } \\
\text { Document Approval }\end{array}$ & 29 \\
\hline $1 \times$ large & Electronic Change Management & 24 \\
\hline
\end{tabular}

\subsection{Method Used to Analyze the Process Models}

To our knowledge there exist no mining techniques to extract activity patterns from real-world process models; i.e., contemporary process mining tools like ProM analyze the event logs (e.g., execution or change logs) related to process execution and do not extract information related to the semantics and the (internal) logic of process activities [14], [5], [15]. Therefore, we perform a manual analysis in order to identify relevant activity patterns as well as their co-occurrences within the 214 process models.

For each workflow activity pattern WAP* $^{*}$ we calculate its support value $S_{W A P^{*}}$, which represents the relative frequency of the respective activity pattern within the set of analyzed process models; i.e., $S_{W A P *}:=$ Freq $\left(W_{A P}^{*}\right) / 214$ where Freq(WAP*) denotes the absolute frequency of WAP* within the collection of the analyzed 214 models; for each process model we count at most one occurrence of a particular pattern.

First, we manually identify and annotate activity patterns in all process models analyzed. Following this, we determine the absolute frequency of each activity pattern WAP $^{*}$ as described above. The obtained results are divided by the total number of analyzed process models (i.e., 214 in our case).

\subsection{Frequency of Activity Patterns in Real-World Process Models}

Our analysis has shown that five out of the seven activity patterns (cf. Table 1) are not dependent on a specific application domain or on a particular organizational structure 
(e.g., the degree of centralization in decision making or the standardization of work abilities). More precisely, this applies to the following five patterns (cf. Table 1): UNidirectional and Bi-Directional PERFORMATIVE (WAP III+IV), DECISION (WAP VII), NOTIFICATION (WAP V), and INFORMATIVE (WAP VI). We could identify these five patterns with high frequency in almost all process models we had analyzed. The latter also applies to the APPROVAL pattern, which can be explained by the high degree of centralization regarding decision-making within the considered organizations (cf. Table 2). This high centralization implies the use of approval activities [16]. By contrast, most of the analyzed process models do not contain QUESTIONRESPONSE activities. Figure 3 graphically illustrates the relative frequency of each activity pattern with respect to the set of analyzed process models.

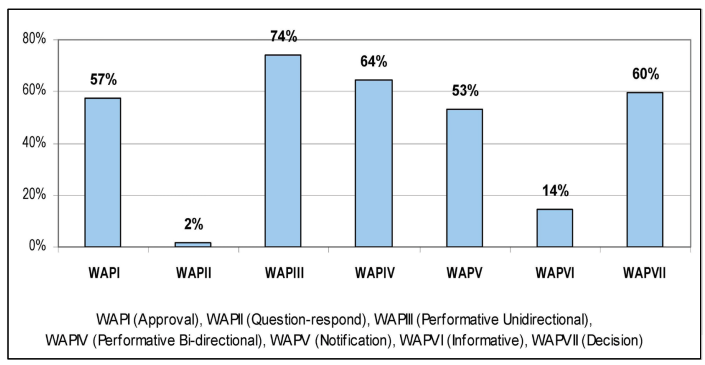

Fig. 3. Frequency of activity patterns in real process models

\subsection{Identifying Co-occurrences of Activity Patterns}

One of the use cases for the ontology of our BPM tool (cf. Section 4) is based on a mechanism that gives design time recommendations with respect to the activity patterns best suited to be combined with the last used pattern. This mechanism utilizes statistical data we gathered during our empirical study. We summarize these statistical findings in this section. To obtain the frequencies for pattern co-occurrences, we analyze the sequences of activity patterns in $154^{1}$ out of 214 studied process models.

Before performing the analysis we classified the business process models into human-oriented models (i.e., processes with human interventions during their execution) and fully automated models (i.e., processes without any human intervention). We verified that certain activity patterns can be find more often in one of the two categories [17]. This analysis has been inspired by a classification provided by Le Chair who distinguishes between system-intensive and human-intensive business processes [18]. System-intensive processes are characterized by being handled on a straight-through basis, i.e., there is minimal or no human intervention during process execution and only few exceptions occur. Human-intensive processes (similar to methods engineering [19]), in turn, require people to get work done by relying on

${ }^{1}$ When performing this analysis we had access to only 154 out of the 214 studied process models. 
business applications, databases, documents, and other people as well as extensive interactions with them. This type of process requires human intuition or judgment for decision-making during individual process steps.

When classifying a subset of the studied process models, for which respective information is available, into these two categories, we obtain 123 human-intensive and 31 system-intensive process models respectively. Note that in this earlier analysis we consider only 154 of the 214 process models studied in total. In a next step we evidence the occurrence of the seven activity patterns with respect to the two categories of process models. Figure 4 shows the support value (i.e., the relative frequency) of the activity patterns in both the system-intensive and the human-intensive process models. As can be seen, some of the patterns (i.e., APPROVAL (WAP I), INFORMATIVE (WAP VI), and QUESTION-RESPONSE (WAP II)) do not appear in system-intensive process models at all. Obviously, these patterns are usually related to human activities; i.e. they are executed by an organizational role.

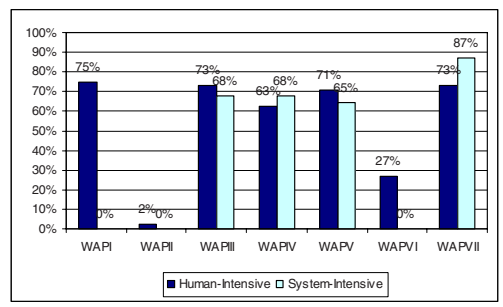

Fig. 4. Frequency of activity patterns in human- and system-intensive process models

In another analysis we have identified frequent and recurrent co-occurrences of activity patterns within process models. Relying on the results of this analysis, our knowledge base and BPM tool respectively display to the process designer a ranking of the activity patterns which most frequently follow the pattern the user applied before during process design. For example, our analysis has shown that the pattern pair DECISION $\rightarrow$ NOTIFICATION occurs more often in system-intensiv than in humanintensive business processes (cf. Fig. 5). Opposed to this, pattern pair DECISION $\rightarrow$ APPROVAL occurs more frequently in human-intensive process models.

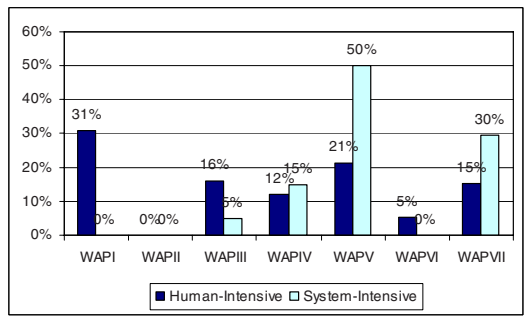

Fig. 5. How often does an activity pattern directly follow the DECISION pattern (regarding both system- and human-intensive processes)? 


\section{Towards a Pattern-Based Process Modeling Tool}

This section presents basic concepts of our BPM tool, which allows to design process models based on the reuse of activity patterns. The latter are described by means of an ontology. In principle, basic concepts behind this BPM tool can be added as extension to existing BPM components as well (e.g., Intalio [20], Aris Toolset [21], or ADEPT Process Composer [22]).

Core functionalities of our BPM tool are as follows:

Assisting users in designing process models. First, the process designer selects the kind of business process (e.g., human intensive) to be modeled, which is then matched to a set of business functions as maintained in the ontology; i.e., the BPM tool adapts a set of business functions to be used for process modeling in the given context. Following this, the process designer chooses a business function and provides contextual data (e.g., about the organization). This information is then matched to an activity pattern as maintained in the aforementioned ontology. Furthermore, the BPM tool recommends to use the respective activity pattern and to apply corresponding design choices; i.e., to configure a concrete pattern variant. Afterwards, the tool recommends the most suitable activity patterns to be used together with the activity pattern applied before. In addition, it informs the user about how frequently each pair of activity pattern (i.e., the previously applied activity pattern plus the recommended activity pattern) was used in earlier modeling. This module is developed based on the analysis results presented in Section 3.3.

Construction of an ontology for activity patterns. The ontology for activity patterns does not only maintain the patterns themselves, but also the frequency with which each pattern has co-occurred with a previously used pattern. Through the analyses of additional process models (e.g., from the automotive as well as the healthcare domain) we aim at increasing the support value of such pairs of activity patterns (cf. Section 3.3). Thus, at design time the pattern pairs being recommended will help to design a process model which is closer to the business process being manually executed in the organization.

\subsection{Architecture of the Process Modeling Tool}

Core components of our BPM Tool are as follows (cf. Fig. 6):

- Query Component: It provides a query mechanism for matching the activity patterns maintained by the ontology with the given kind of business process (e.g., human intensive), business function (e.g., approval), organizational context (e.g., level of centralization in decision-making), and corresponding design choice as chosen by the user (if not set automatically).

- Ontology Manager: It comprises an ontology and a query mechanism (Business Function Query + WAP Query). The ontology describes the activity patterns (cf. Fig. 6) and their properties (e.g., attributes and relationships with other patterns). Our query as well as update mechanisms give design time recommendations with respect to the most suited activity patterns to be combined with an already used one. An example of a query would be the selection of the 
business functions which occur more frequently in system-intensive process models. In addition, our update mechanism has to be used to adapt relative frequency of each pattern pair (e.g., based on the analysis of new process models) as identified in our process model analysis.

- Scheme Translation: This component is responsible for translating a process model (based on translation algorithms) which uses activity patterns as building blocks (stored in XML code) to either a conventional notation (e.g., BPMN) or an existing process execution language (e.g., BPEL, XPDL). The use of this translation component is optional, i.e., it will be only applicable if the user wants the respective process model being translated to another notation and process execution language respectively.

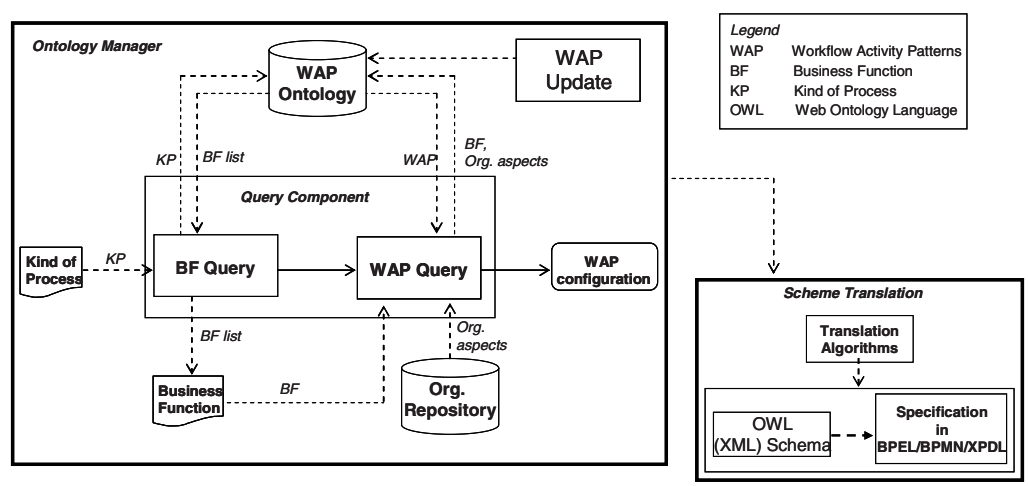

Fig. 6. Architecture of the ProWAP process modeling tool

\subsection{Interacting with the Process Modeling Tool}

We sketch basic steps of our pattern-based modeling approach: First, the user specifies the kind of process to be designed (e.g., system- vs. human-intensive) (cf. Step 1 in Fig. 7). Taking this information, in Step 2 the BPM tool presents a set of business functions relevant in the given context (cf. Section 3.2). In Step 3, the user selects a business function (e.g., approval) and additionally specifies information about the organizational context within which this function is executed (e.g., organizational unit with centralized decision-making).

Based on this information and on the defined ontology, the best suited activity pattern (incl. corresponding design choices) is queried (cf. Step 4 in Fig. 7). More precisely, the query results in an instance of an activity pattern and a set of related attributes (e.g., kind of approval, list of reviewers in case of concurrent or iterative approvals, application-specific details about the approval like approval conditions, etc.). In Step 5, the user then customizes the activity pattern to the given context (e.g., kind of approval (iterative approval by a hierarchy of organizational roles); list of reviewers (\#id): 101, 106, 200; Approval activity description: paper review; conditions to approve: at least 2 strong acceptances). In Step 6, the BPM tool creates an instance of the corresponding pattern according to this customization. Afterwards, the BPM tool recommends a list of activity patterns (with corresponding statistics regarding the use 


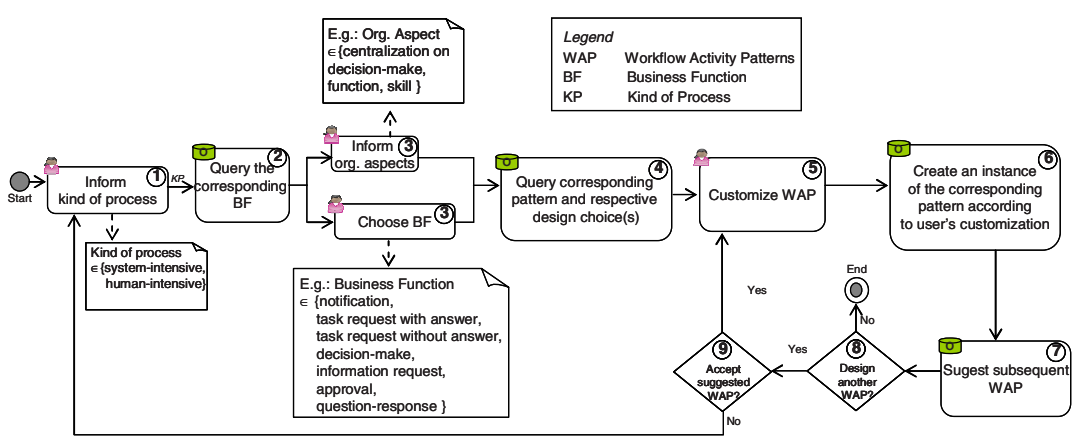

Fig. 7. Procedure showing how the user interacts with the BPM tool

of these patterns in other process models) to follow the previously modeled activity (cf. Step 7 in Fig. 7). For example, APPROVAL is followed by NOTIFICATION in $24 \%$ of the 154 process models we analyzed. The user must then state whether another activity pattern shall be designed and whether the pattern suggested by the BPM tool shall be used (cf. Steps 8 and 9).

\subsection{Ontology of Workflow Activity Patterns}

We now introduce our ontology for workflow activity patterns (cf. Fig.8). Particularly, this ontology has been used when implementing the recommendation mechanism of our BPM Tool. The ontology represents the structure of the activity patterns, related design choices (e.g., single and iterative approval), and their relationships. Altogether, the main goal of this ontology is to better define the structure of activity patterns, their attributes, and their relationships. In addition, the ontology maintains the use statistics for each activity pattern (in the context of process modeling) as well as the co-occurrences of pattern pairs based on the empirical study reported in Section 3.3. Our ontology also integrates information about the organizational context in which the activity patterns are usually applied. Such information is matched with the business function to identify the most suitable activity patterns to be used together with the activity pattern designed before.

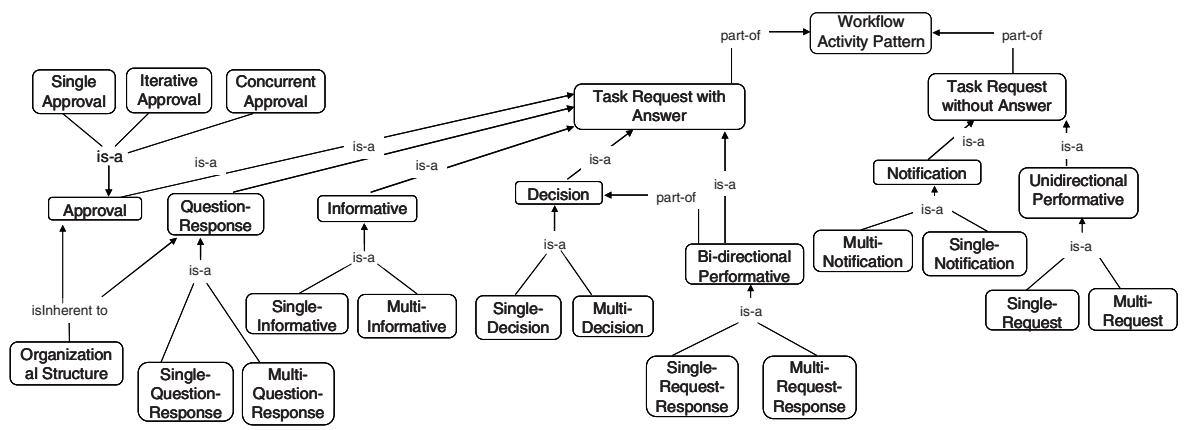

Fig. 8. Ontology of Workflow Activity Patterns (simplified and partial views) 
The diagram depicted in Fig. 8 represents the Workflow Activity Patterns ontology (partial view). It describes the taxonomy of the activity patterns, i.e. a conceptual hierarchical structure that relates classes and sub-classes to each other. The most general class is WORKFLOW ACTIVITY PATTERN. This concept has two main components: TASK REQUEST WITH ANSWER and TASK REQUEST WITHOUT ANSWER. The first component is composed by the following concepts, which represent the Workflow Activity Patterns: APPROVAL, QUESTION-RESPONSE, INFORMATIVE, DECISION and BIDiRECTIONAL PERformative. The TASK REQUEST Without AnSWER concept is composed by the two patterns NOTIFICATION and UNIDIRECTIONAL PERFORMATIVE.

The APPROVAL and QUESTION-RESPOND patterns are related to organizational structure aspects. In the context of an APPROVAL pattern, an object approval is always performed by specific roles inside an organization. The QUESTION-RESPONSE pattern is applied when an actor might have a question in order to work on the process or on a particular process activity. The question is forwarded to a specific organizational role that has the appropriate expertise to answer it.

As aforementioned each workflow activity pattern has specific design choices. For example, the design choices of task execution pattern allow expressing at design time if the task execution is requested from a single actor or from multiple actors. In Fig. 8 such variants are represented as subclasses of the pattern concepts, i.e. as specializations. The Approval concept, for example, has three specializations: Single Approval, Iterative Approval and Concurrent Approval. They represent the Approval pattern design choices. Single Approval is associated with exactly one reviewer. Iterative Approval is handled by a loop based on a list of reviewers. This list can be often related to vertical organizations where there is a hierarchical organizational structure. Regarding Concurrent Approval, the approval request is sent to the whole group of reviewers simultaneously. The final decision is then made after all reviewers have performed their evaluation.

Our ontology (complete view) also considers system- and human-intensive processes. In addition, we have defined attributes for each class and sub-class of our ontology; e.g., way of notification (e.g., by e-mail or as work item in the participants' worklists), organizational role, and activity status.

\section{Related Work}

Recently, numerous approaches on workflow patterns have been proposed to support both the conceptual and the implementation level of business processes. One of the first contributions in this respect was a set of process patterns to be used in the context of software processes within an organization [23].

Russell proposes 43 workflow patterns for describing process behavior [24], [5]. Each pattern represents a routing element (e.g., sequential, parallel and conditional routing) which can be used in process definitions. In the meantime these workflow patterns have been additionally used for evaluating process specification languages and process modeling tools [25], [26].

A set of data patterns is proposed by [7]. These patterns are based on data characteristics that occur repeatedly in different workflow modeling paradigms. Examples are data visibility and data interaction. In another work, Russell presents a set of 
resource patterns of which each one describes a way through which resources can be represented and utilized in process models [6]. A resource is considered as an entity capable of doing work. It can be either human (e.g., a worker) or non-human (e.g., equipment). Examples of resource patterns include Direct Allocation and Role-Based Allocation. Finally, process change patterns and change support features have emerged to effectively deal with (dynamic) process changes [11], [30].

Recently, Russell has presented a pattern-based classification framework for characterizing exception handling in workflow management systems [8]. This framework has been used to examine the capabilities of workflow management and BPM systems and to evaluate process specification as well as process execution languages. As a result, the authors emphasis the limited support for exception handling in existing workflow management systems.

Barros proposes a set of service interaction patterns, which allow for service interactions, pertaining to choreography and orchestration, to be benchmarked against abstracted forms of representative scenarios [10]. As example consider the Send pattern and the Send/Receive pattern. Altogether Russell and Barros provide a thorough examination of the various perspectives that need to be supported by a workflow language and BPM tool respectively. However, none of these approaches investigate which are the most frequent patterns recurrently used during process modeling and in which way the introduction of such activity patterns eases process modeling.

SAP has developed a cross-application engine called SAP Business Workflow [27]. This tool enables the process-oriented integration of business objects and applications including a workflow wizard with workflow templates and process reference models. Related to that is the Supply-Chain Operations Reference-model (SCOR) which is based on best practices as well [28].

Finally, PICTURE proposes a set of 37 domain-specific process building blocks. More precisely, these building blocks are used by end users in public administrations to capture and model the process landscape. The building blocks have been evaluated in practice [29].

\section{Summary and Outlook}

In this paper, we identified seven workflow activity patterns necessary and in many cases also sufficient to model a large variety of process models. Moreover we discussed results of an empirical study where we had analyzed how often activity patterns as well as co-occurrences of them (i.e. pairs of activity patterns) are present in a large collection of real process models.

We additionally proposed an approach for process modeling based on workflow activity patterns. Basic to this tool is the reuse of the presented activity patterns. Respective functionality can be added as extension to existing process modeling components as well. Our goal is to increase the reuse of recurrent business functions and to better assist users in the design of high-quality process models; i.e., by providing context-specific recommendations on which patterns to use for process modeling next. It is important to mention that our patterns can be used together with other patterns related to control flow [5] or process change [11], [30]. In this paper we have given first insights into the architecture of our BPM tool as it is implemented in the ProWAP project. 
The main advantages of our approach can be summarized as follows: (a) the completeness and necessity of the activity patterns for process design have been empirically evidenced; (b) a small set of parameterizable activity patterns is sufficient to model a large variety of processes, which reduces the complexity with respect to pattern usage; (c) the concepts realized in our process modeler are tool-independent and can be adapted for any process modeling tool; and (d) the sketched recommendation mechanisms can be useful to reduce complexity in process design as well as to improve semantical model correctness.

In future work, we intend to identify variants of each pattern concerning specific application domains. For example, we want to figure out what kind of approvals occur most frequently in the healthcare and in the automotive domain. Furthermore, we will perform additional analyses considering process models from different application domains (e.g., health insurance and automotive engineering). Our goal is to identify more common occurrences of pairs of activity patterns. We also aim at extending the tool with a mechanism to learn from designer actions. Last but not least, we intend to investigate how to transform process models defined with our tool (and being based on the activity patterns) into conventional notations and languages respectively.

\section{Acknowledgements}

The authors would like to acknowledge the Coordination for the Improvement of Graduated students (CAPES), the Institute of Databases and Information Systems of the University of Ulm (Germany), and the Informatics Institute of Federal University of Rio Grande do Sul (Brazil).

\section{References}

1. Rummler, G., Brache, A.: Improving Performance: How to Manage the White Space on Organizational Chart. Jossey-Bass, San Francisco (1990)

2. Lenz, R., Reichert, M.: IT Support for Healthcare Processes - Premises, Challenges, Perspectives. Data and Knowledge Engineering 61, 39-58 (2007)

3. Thom, L., Iochpe, C., Amaral, V., Viero, D.: Toward Block Activity Patterns for Reuse in Workflow Design. In: Workflow Handbook 2006, pp. 249-260 (2006)

4. Mutschler, B., Reichert, M., Bumiller, J.: Unleashing the Effectiveness of Process-oriented Information Systems: Problem Analysis, Critical Success Factors and Implications. IEEE Transactions on Systems, Man, and Cybernetics (Part C) 38(3), 280-291 (2008)

5. van der Aalst, W.M.P., ter Hofstede, A.H.M., Kiepuszewski, B., Barros, A.: Workflow Patterns. Distributed and Parallel Databases 14(3), 5-51 (2003)

6. Russel, N., Aalst, W., Hofstede, A., Edmond, D.: Workflow Resource Patterns: Identification, Representation and Tool Support. In: Pastor, Ó., Falcão e Cunha, J. (eds.) CAiSE 2005. LNCS, vol. 3520, pp. 216-232. Springer, Heidelberg (2005)

7. Russel, N., Hofstede, A., Edmond, D.: Workflow Data Patterns. In: Delcambre, L.M.L., Kop, C., Mayr, H.C., Mylopoulos, J., Pastor, Ó. (eds.) ER 2005. LNCS, vol. 3716, pp. 353-368. Springer, Heidelberg (2005)

8. Russel, N., Aalst, W., Hofstede, A.: Workflow Exception Patterns. In: Dubois, E., Pohl, K. (eds.) CAiSE 2006. LNCS, vol. 4001, pp. 288-302. Springer, Heidelberg (2006)

9. Ohnmacht, A.: Development of a Collaborative Process Modeling Methodology for Domain Experts. Master Thesis, University of Ulm (2007) 
10. Barros, A., Dumas, M., ter Hofstede, A.: Service Interaction Patterns. In: van der Aalst, W.M.P., Benatallah, B., Casati, F., Curbera, F. (eds.) BPM 2005. LNCS, vol. 3649, pp. 302-318. Springer, Heidelberg (2005)

11. Weber, B., Rinderle, S., Reichert, M.: Change Patterns and Change Support Features in Process-Aware Information Systems. In: Krogstie, J., Opdahl, A., Sindre, G. (eds.) CAiSE 2007. LNCS, vol. 4495, pp. 574-588. Springer, Heidelberg (2007)

12. Namiri, K., Stoganovic, N.: Pattern-Based Design and Validation of Business Process Compliance. In: Proc. CoopIS 2007, pp. 59-76 (2007)

13. Thom, L.: Applying Block Activity Patterns in Workflow Modeling. In: Proc. 8th Int'1 Conf. on Enterprise Information Systems (ICEIS 2006), Paphos, Cyprus, pp. 457-460 (2006)

14. Ellis, C.: Workflow Mining: Definitions, Techniques, Future Directions. In: Fischer, L. (ed.) Workflow Handbook 2006. Lighthouse Point: Future Strategies, pp. 213-228 (2006)

15. Günther, C.W., Rinderle-Ma, S., Reichert, M., van der Aalst, W.M.P., Recker, J.: Using Process Mining to Learn from Process Changes in Evolutionary Systems. Int'l Journal of Business Process Integration and Management (2008)

16. Davis, M.R., Weckler, D.A.: A Practical Guide to Organization Design. Crisp Publications, Boston (1996)

17. Chiao, C., Thom, L.H., Iochpe, C., Reichert, M.: Verifying Existence, Completeness and Sequences of Workflow Activity Patterns in Real Process Models. In: IV Brazilian Symposium of Information Systems (SBSI), Rio de Janeiro, Brazil (2008)

18. Le Clair, C., Teubner, C.: The Forrester Wave: Business Process Management for Document Processes, Q3 (2007)

19. Wiley, J.: Methods Engineering, United States of America (1962)

20. Intalio. Creating Process Flows (2006), http://bpms.intalio.com

21. IDS Scheer: Aris Platform: Product Brochure (2007), http://www.idsscheer.com/set/82/PR_09-07_en.pdf

22. Reichert, M., Rinderle, S., Kreher, U., Dadam, P.: Adaptive Process Management with ADEPT2. In: Proc. Int'l Conf. on Data Engineering (ICDE 2005), Tokyo, Japan, pp. 1113-1114. IEEE Computer Society Press, Los Alamitos (2005)

23. Ambler, S.W.: An Introduction to Process Patterns (1998)

24. Russell, N., Hofstede, A.H.M., ter Aalst, W.M.P., van der Mulyar, N.: Workflow Control Flow Patterns: A Revised View. BPM Center Report BPM-06-22, BPM center.org (2006)

25. van der Aalst, W.M.P.: Patterns and XPDL: A Critical Evaluation of the XML Process Definition Language. QUT Technical report, FIT-TR-2003-06, Queensland University of Technology, Brisbane (2003)

26. Wohed, P., Aalst, W.M.P., van der Dumas, M., ter Hofstede, A.H.M., Russell, N.: Patternbased Analysis of BPMN - An extensive evaluation of the Control-flow, the Data and the Resource Perspectives. BPM Center Report BPM-06-17, BPMcenter.org (2006)

27. SAP. SAP Business Workflow (2008), http://www.sap.com

28. SCOR. Supply-Chain Operations Reference-model (2008)

29. Becker, J., Pfeiffer, D., Räckers, M.: Domain Specific Process Modelling in Public Administrations - The PICTURE-Approach. In: Wimmer, M.A., Scholl, J., Grönlund, Å. (eds.) EGOV. LNCS, vol. 4656, pp. 68-79. Springer, Heidelberg (2007)

30. Weber, B., Reichert, M., Rinderle-Ma, S.: Change Patterns and Change Support Features Enhancing Flexibility in Process-Aware Information Systems. Data and Knowledge Engineering (accepted for publication, 2008) 